\title{
THE POSSIBILITY OF SUPERSONIC PLASMA FLOW IN A COLLAPSING POST-SUNSET IONOSPHERE
}

\author{
ERNEST G. FONTHEIM \\ Space Physics Research Laboratory, Department of Electrical Engineering, University of \\ Michigan, Ann Arbor, Michigan 48105, U.S.A. \\ and

\section{P. M. BANKS} \\ Department of Applied Physics and Information Science, University of California at San Diego, \\ La Jolla, California 92037, U.S.A.
}

(Received 10 August 1971)

\begin{abstract}
As a result of the rapidly decreasing pressure in the topside ionosphere during twilight hours, a rapid downward flow of hydrogen plasma from the protonosphere takes place. In the case of steady state, isothermal, frictionless flow the criterion for the existence of a critical point (transition to supersonic flow) above $1000 \mathrm{~km}$ is that the plasma temperature be lower than a certain limiting temperature $T_{l}$ which is a function of the field line considered. In the latitude region between $40^{\circ}$ and $70^{\circ}$ this upper temperature limit varies from $963^{\circ} \mathrm{K}$ to $1066^{\circ} \mathrm{K}$. Since these temperatures are considerably lower than the observed temperatures, it follows that in the case of steady state, isothermal flow the velocities will always remain subsonic. When the effect of the neglected terms is examined, the temperature gradient is shown to exert the strongest influence on the nature of the flow. For each latitude there is shown to exist a certain gradient $(\partial T / \partial r)_{0}$ such that, if $\partial T / \partial r<(\partial T / \partial r)_{0}$, the criterion for a critical point to exist above $1000 \mathrm{~km}$ is again that the temperature at the critical point be less than some limiting temperature $T_{l}$. If, however, $\partial T / \partial r>(\partial T / \partial r)_{0}$, then the criterion turns out to be that the temperature at the critical point be larger than $T_{\ell}$. The values of $(\partial T / \partial r)_{0}$ are between $9 \times 10^{-6}{ }^{\circ} \mathrm{K} \mathrm{cm}{ }^{-1}$ and $6.75 \times 10^{-6}{ }^{\circ} \mathrm{K} \mathrm{cm}^{-1}$ for latitudes between $40^{\circ}$ and $70^{\circ}$. For values of the temperature gradient above about $4 \times 10^{-6}{ }^{\circ} \mathrm{K} \mathrm{cm}^{-1}$ the criterion is satisfied for physically realistic temperatures (above $1500^{\circ} \mathrm{K}$ ), i.e. a critical point may exist above $1000 \mathrm{~km}$. On this basis it is concluded that there is a definite possibility that supersonic downward flows in a post-sunset topside ionosphere may occur.
\end{abstract}

\section{INTRODUCTION}

Several years ago Nagy et al. (1968) (henceforth referred to as NBF) calculated electron density and temperature profiles for nighttime conditions at mid-geomagnetic latitudes. Their results indicated that during early evening hours the plasma contained in field tubes near $50^{\circ}$ magnetic latitude attained supersonic speeds $\left[M\left(\mathrm{H}^{+}\right)>1\right.$ where $M=u / c, c^{2}=$ $\left.2 \mathrm{kT} / \mathrm{m}\left(\mathrm{H}^{+}\right)\right]$as a result of rapidly decreasing plasma pressure at their $1000 \mathrm{~km}$ lower altitude boundary. It was noted, however, that the calculations were based on a diffusion approximation to the ion momentum equation with the terms $\partial u / \partial t$ and $u \partial u / \partial s$ neglected. As a consequence the plasma flow speed was not accurately described as it approached the ion sound speed, and the calculated transition to supersonic flow did not represent a true critical point.

In this article we consider the problem of supersonic flow in a collapsing night-time topside ionosphere in more detail. Unfortunately, the problem of the time dependent evacuation of plasma from a field tube is sufficiently difficult to preclude simple numerical solutions. We, therefore, give the arguments relating to the possible existence of a critical point. No computations of density, flow speed, or temperature are attempted.

The essential idea behind supersonic flow in a collapsing night-time ionosphere is that gravitational potential energy of plasma at altitudes of 3-4 earth radii can be converted into kinetic energy of directed motion during twilight hours when the plasma pressure in the 


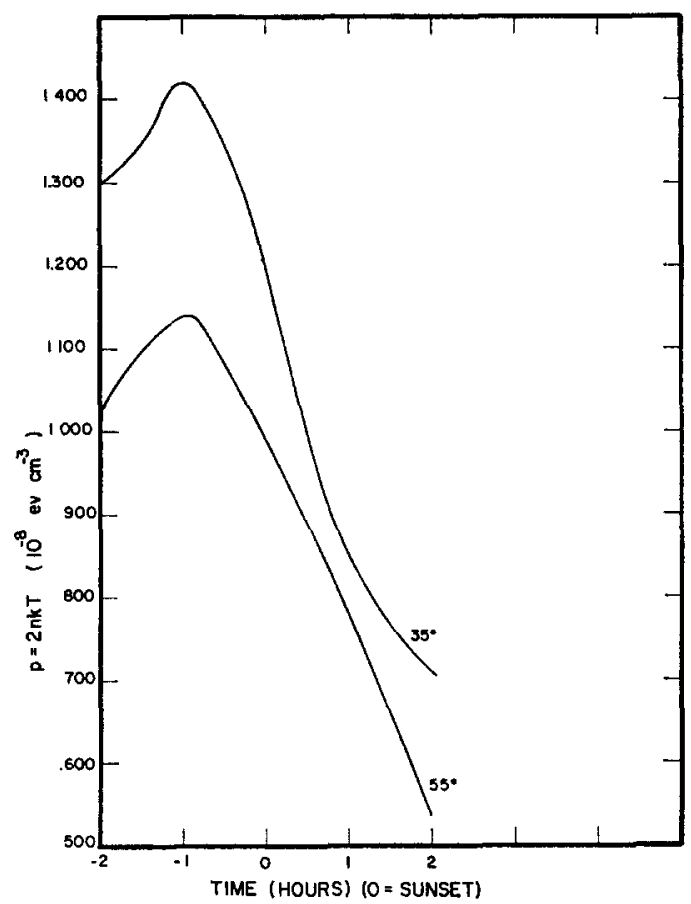

Fig. 1. RAPID PRESSURE DECREASE AFTER SUNSET IN THE TOPSIDE IONOSPHERE (BASED ON DATA BY BRACE et al., 1967).

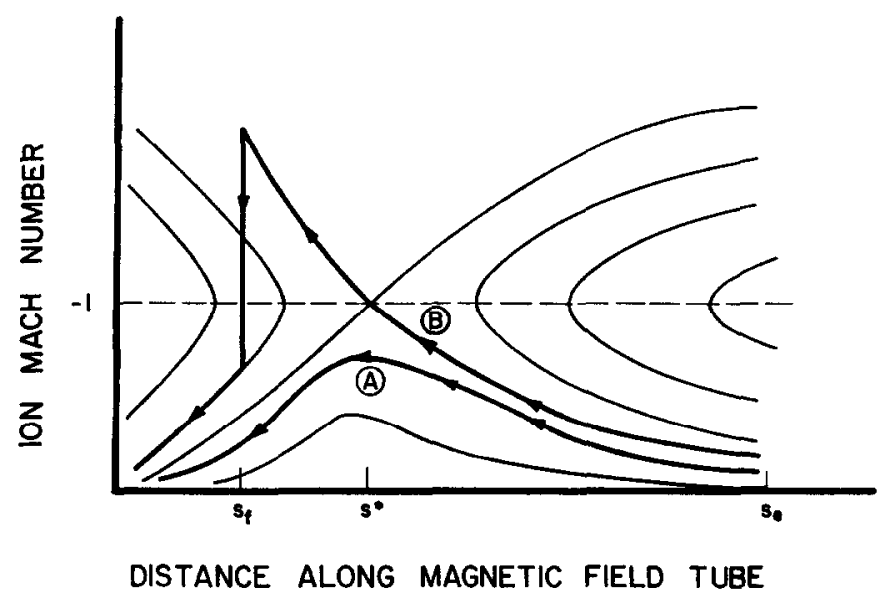

Fig. 2. FAMILY OF SOLUTIONS OF THE ION TRANSPORT EQUATION FOR INWARD FLOW $(M<0)$. $s_{e}$ is the coordinate of the equatorial plane, $s^{*}$ is the coordinate of the critical point, and $s_{\text {, }}$ that of the shock front.

topsideionosphere decreases rapidly (see Fig. 1). If the inward plasma flow remains subsonic along the field tube, the ion kinetic energy of directed motion is gradually converted to random thermal energy through ion-neutral collisions and compressive heating. Such a feature was clearly shown by the results of NBF. If the flow becomes supersonic, however, most of the excess ion kinetic energy is released to ion thermal energy at a shock front where the downward supersonic flow becomes subsonic. Figure 2 schematically illustrates the 
different solutions to the ion transport equation. Here $s$ is a coordinate along the field line and the Mach number, $M$, is negative for inward flow. One of the many subsonic solutions is shown by curve $A$, while the inward supersonic solution is given by curve $B$. The critical point occurs at the point $s^{*}$, while the shock front and transition to subsonic flow is found at $s_{f}$.

\section{CRITERION FOR SUPERSONIC FLOW}

The time-dependent equations of continuity, momentum, and energy for the topside hydrogen plasma are (see e.g. Banks and Holzer, 1969)

$$
\begin{gathered}
\frac{\partial n}{\partial t}+n M c\left[\frac{1}{n} \frac{\partial n}{\partial s}+\frac{1}{M} \frac{\partial M}{\partial s}+\frac{1}{c} \frac{\partial c}{\partial s}+\frac{1}{S} \frac{\partial S}{\partial s}\right]=0 \\
\frac{1}{c} \frac{\partial M}{\partial t}+M \frac{\partial M}{\partial s}+\frac{1}{n} \frac{\partial n}{\partial s}+\frac{M}{c^{2}} \frac{\partial c}{\partial t}+\frac{1}{c}\left(M^{2}+2\right) \frac{\partial c}{\partial s}+\frac{1}{c^{2}} \frac{\partial \phi}{\partial s}=-\frac{M}{c} v_{\text {in }} \\
3 n k \frac{\partial T}{\partial t}+3 n M c k \frac{\partial T}{\partial s}-\frac{1}{S} \frac{\partial}{\partial s}\left(S K T^{5 / 2} \frac{\partial T}{\partial s}\right)+\frac{2 n k T}{S} \frac{\partial}{\partial s}(S M c)-\mu n M^{2} c^{2} v_{\text {in }}=-L
\end{gathered}
$$

where

$$
\begin{aligned}
n= & \text { number density of hydrogen ions } \\
M= & u / c=\text { ion Mach number (positive for outward flow) } \\
c^{2}= & 2 \mathrm{kT} / \mathrm{m} \\
T=T_{i}=T_{b}= & \text { electron (ion) temperature } \\
m= & \text { proton mass } \\
s= & \text { coordinate along the magnetic field line (positive in the outward } \\
& \text { direction) } \\
v_{\text {tn }}= & \text { ion-neutral collision frequency } \\
= & 9 \cdot 5 \times 10^{-13} n(\mathrm{H})\left(T+T_{n}\right)^{1 / 2}\left[14 \cdot 4-1 \cdot 15 \log \left(T+T_{n}\right)\right]^{2} \mathrm{sec}^{-1} \\
& (\text { Banks, } 1966) \\
n(\mathrm{H})= & \text { hydrogen density } \\
T_{n}= & \text { neutral temperature } \\
K T^{5 / 2}= & \text { electron thermal conductivity } \\
= & 7 \cdot 7 \times 10^{5} T^{5 / 2} \mathrm{eV} \mathrm{cm} \mathrm{cm}^{-1} \mathrm{sec}^{-1}\left({ }^{\circ} \mathrm{K}\right)^{-1} \\
L= & \text { ion-neutral loss rate } \\
= & 1 \cdot 4 \times 10^{-14} n n(\mathrm{H})\left(T+T_{n}\right)^{1 / 2}\left(T-T_{n}\right) \mathrm{ev} \mathrm{cm}^{-3} \mathrm{sec}^{-1} \\
& (\text { Banks, } 1966) \\
\phi= & \text { gravitational potential } \\
S= & \text { cross-sectional area of the magnetic field tube. }
\end{aligned}
$$

Combining Equations (1) and (2) one obtains

$$
\begin{aligned}
\frac{1}{M} \frac{\partial M}{\partial s}\left(M^{2}-1\right)=-\frac{1}{c^{2}} \frac{\partial \phi}{\partial s}+\frac{1}{S} \frac{\partial S}{\partial s}-\frac{M}{c} v_{\text {in }} & +\frac{1}{n M c} \frac{\partial n}{\partial t} \\
& -\frac{1}{c} \frac{\partial M}{\partial t}-\frac{1}{c} \frac{\partial c}{\partial s}\left(M^{2}+1\right)-\frac{M}{c^{2}} \frac{\partial c}{\partial t} .
\end{aligned}
$$


Steady state isothermal case

For time-independent conditions

$$
\left(\frac{\partial n}{\partial t}=\frac{\partial M}{\partial t}=\frac{\partial T}{\partial t}=0\right)
$$

the location of the critical point for inward flow is determined by the roots of the right-hand side of Equation (4) with $M=-1$. Hence, the location of the critical point, $s^{*}$, in the time-independent case, is obtained by the solution of the equation

$$
\frac{1}{c^{2}} \frac{\partial \phi}{\partial s}=-\frac{2}{c} \frac{\partial c}{\partial s}+\frac{1}{S} \frac{\partial S}{\partial s}+\frac{\nu_{\text {in }}}{c} .
$$

Before considering the general solution of Equation (5) including height dependent temperatures, it is instructive to discuss the isothermal case. In addition, we let $v_{\text {in }}=0$ since in most situations the $\mathrm{H}^{+}-\mathrm{H}$ collision frequency does not play an important part in determining the location of $s^{*}$ at high altitudes. The critical point is then determined by the equation

$$
\frac{1}{c^{2}} \frac{\partial \phi}{\partial s}=\frac{1}{S} \frac{\partial S}{\partial s}
$$

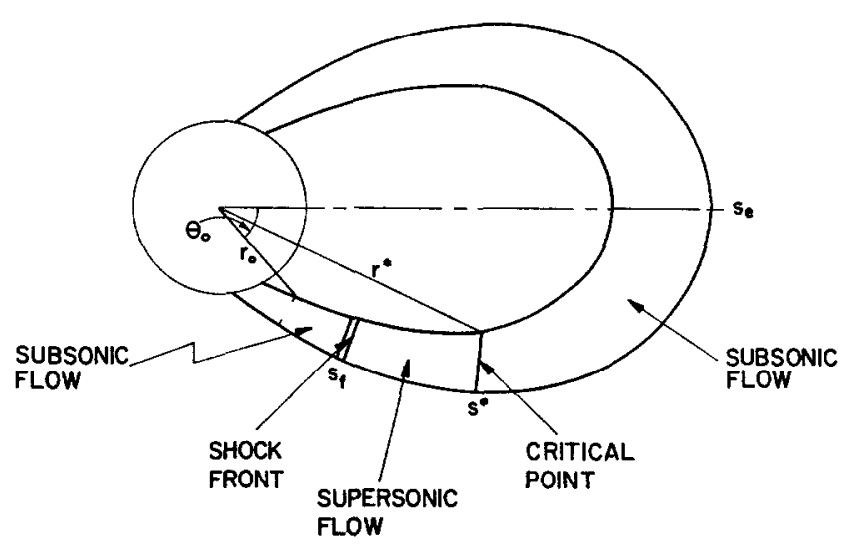

Fig. 3. MAGNETIC FIELD TUBE WITH DIfFeRENT FLOW REGIMES.

$r_{0}$ is the radial distance to the point at $1000 \mathrm{~km}$ altitude.

An explicit relation can be obtained for Equation (6) with the assumption of a dipole magnetic field. Using the definitions of Fig. 3 we obtain the relation

$$
\frac{r^{*}}{r_{0}}=\frac{2 g_{0} r_{0}\left(4-3 \cos ^{2} \theta_{0} \frac{r^{*}}{r_{0}}\right)}{3 c^{2}\left(8-5 \cos ^{2} \theta_{0} \frac{r^{*}}{r_{0}}\right)}
$$

where the radius vector $r^{*}$ has been used rather than $s^{*}$, the coordinate along the field line and where $r_{0}$ is the lower limit below which $\mathrm{H}^{+}$is no longer the major ion, and $\theta_{0}$ is the latitude at $r_{0}$. This defines the field tube under consideration. $g_{0}$ is the gravitational acceleration at $r_{0}$. The ratio $r^{*} / r_{0}$ must be larger than unity in order for the critical point to lie 
above $r_{0}$. (Below $r_{0} \mathrm{H}^{+}$is no longer the sole constituent, and Equations (1)-(3) must be replaced by a set of equations including the presence of $\mathrm{O}^{+}$which has the effect of slowing down the flow.) Hence, the criterion for supersonic flow to exist is that $r^{*} / r_{0}>1$. Equation (7) can be solved for $r^{*} / r_{0}$. The result is:

$$
\frac{r^{*}}{r_{0}}=\frac{6\left(g_{0} r_{0} \cos ^{2} \theta_{0}+4 c^{2}\right) \pm\left[36 g_{0}^{2} r_{0}^{2} \cos ^{4} \theta_{0}+\left(24 c^{2}\right)^{2}+48 g_{0} r_{0} c^{2} \cos ^{2} \theta_{0}\right]^{1 / 2}}{30 c^{2} \cos ^{2} \theta_{0}} \text {. }
$$

It can be easily shown that the solution with the positive square root is unphysical since it corresponds to a value of $r^{*}$ which is larger than the equatorial radius of the field line. The inequality $r^{*} / r_{0}>1$ is satisfied if the plasma temperature lies below some limiting value $T_{l}^{\circ}$ which is a function of the field line under consideration. If $r_{0}=7370 \mathrm{~km}(1000 \mathrm{~km}$ altitude), the values of $T_{l}^{\circ}$ for the field lines of interest here are given in Table 1 below.

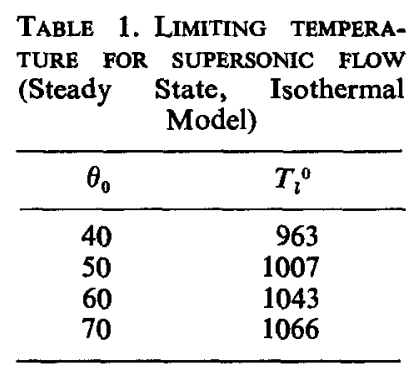

Here $\theta_{0}$ is the latitude of the $1000 \mathrm{~km}$ altitude point of the field line. Hence, in the case of frictionless isothermal flow a steady state critical point will be found above $1000 \mathrm{~km}$ only if $T<T_{l}^{\circ}$. These temperatures are too low for immediate post-sunset conditions at those latitudes. Therefore it must be concluded that for the case of steady state isothermal flow the velocities will always be subsonic.

\section{General case}

Equation (6) and the above discussion ignore the time-dependent nature of the collapsing topside ionosphere as well as the temperature variation along the field line and ion-neutral friction. It is of interest to investigate the effect of those mechanisms on the location of the critical point. Setting the right-hand side of Equation (4) equal to zero with $M=-1$ and again using a dipole magnetic field one obtains the expression

$$
\frac{3}{2 r_{0} x} \frac{8-5 x \cos ^{2} \theta_{0}}{4-3 x \cos ^{2} \theta_{0}} c^{2}+\left(v_{\mathrm{iL}}-\frac{1}{n} \frac{\partial n}{\partial t}-\frac{\partial M}{\partial t}\right) c-\frac{g_{0}}{x^{2}}-\frac{2 k}{m} \frac{\partial T}{\partial r}-M \frac{\partial c}{\partial t}=0,
$$

where $x \equiv r^{*} / r_{0}$. Equation (9) represents the exact relation [in the sense of Equations (1)(3)] between the various plasma parameters at the critical point. For realistic values of the variables the last term on the left-hand side is at least two orders of magnitude smaller than the other terms and will therefore be neglected. Without knowledge of the analytical form of the remaining terms it is only possible to estimate their effect on the temperature limit by using observed numerical values for $n, \partial n / \partial t, \partial M / \partial t$, and $\partial T / \partial r$. If one lets $x=1$, then Equation (9) is a quadratic equation in the ion thermal velocity $c$ at the critical point. For an altitude of $1000 \mathrm{~km}$ and a time shortly after sunset at equinox the following numerical values (based on NBF and the results of Brace et al., 1967) are being used in solving 
Equation (9): $v_{\mathrm{fn}}=2.5 \times 10^{-4} \mathrm{sec}^{-1}, n=1.3 \times 10^{4} \mathrm{~cm}^{-3}, \quad \partial n / \partial t=-0.75 \mathrm{~cm}^{-3} \mathrm{sec}^{-1}$, and $\partial M / \partial t=-3 \times 10^{-4} \mathrm{sec}^{-1}$.

Since the solution of Equation (9) is relatively insensitive to changes in the value of the factor of $c$ and since all of the parameters listed above appear only in that factor, the temperature limit is essentially constant over reasonable ranges of those parameters. This has been verified by numerical checks. The value of the temperature gradient $\partial T / \partial r$, on the other hand, strongly controls the solution of Equation (9). In fact, a positive temperature gradient has the effect of a downward force, as can be seen from Equation (4). Therefore, Equation (9) has been solved for a series of values of $\partial T / \partial r$ between 0 and $10^{-5}{ }^{\circ} \mathrm{K} \mathrm{cm}^{-1}$ and for the fixed values of the other parameters given above. The results of these calculations are presented in Fig. 4.

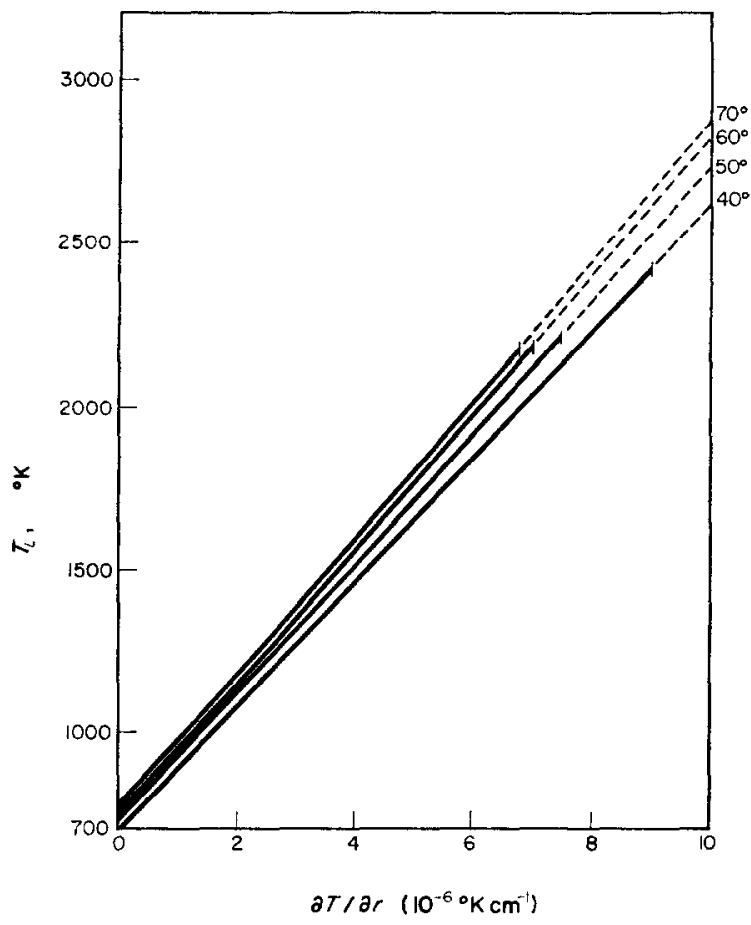

FIG. 4. LIMITING TEMPERATURE FOR SUPERSONIC FLOW AS A FUNCTION OF TEMPERATURE GRADIENT AT FOUR DIFFERENT LATITUDES.

The solid sections represent an upper temperature limit and the dashed sections a lower limit.

\section{DISCUSSION}

The most interesting result of these calculations is that if the temperature gradient is below a certain value $(\partial T / \partial r)_{0}$, then the limiting temperature $T_{l}$ represents an upper limit (just as in the isothermal case), but if $\partial T / \partial r>(\partial T / \partial r)_{0}$, then $T_{l}$ is a lower limit. Thus, if $\partial T / \partial r>(\partial T / \partial r)_{0}$, the plasma temperature at the critical point must be larger than $T_{l}$ in order for the critical point to lie above $r_{0}$. The value of $(\partial T / \partial r)_{0}$ is a function of the latitude. The sections of the curves representing an upper temperature limit $\left[\partial T / \partial r<(\partial T / \partial r)_{0}\right]$ are shown solid in Fig. 4 and those representing a lower limit $\left[\partial T / \partial r>(\partial T / \partial r)_{0}\right]$ are dashed. The values of $(\partial T / \partial r)_{0}$ at four different latitudes are given in Table 2. 
TABLE 2. VALUES OF THE TEMPERATURE GRADIENT AT WHICH $T_{l}$ CHANGES FROM UPPER LIMIT TO LOWER LIMIT

\begin{tabular}{cc}
\hline$\theta$ & \multicolumn{1}{c}{$(\partial T / \partial r)_{0}$} \\
\hline $40^{\circ}$ & $9.00 \times 10^{-6} \mathrm{Kcm}^{-1}$ \\
$50^{\circ}$ & $7.61 \times 10^{-6}$ \\
$60^{\circ}$ & $7.02 \times 10^{-6}$ \\
$70^{\circ}$ & $6.75 \times 10^{-6}$ \\
\hline
\end{tabular}

It must, of course, be kept in mind that these values depend on the numerical values of the other parameters in Equation (9). However, for realistic ranges of those variables $(\partial T / \partial r)_{0}$ changes only very little.

If for a given value of $\partial T / \partial r$ the plasma temperature at some latitude lies either above the solid section or below the dashed section, then there will not be a critical point above $1000 \mathrm{~km}$, and the flow remains subsonic because of the rapidly increasing $\mathrm{O}^{+}$density below $1000 \mathrm{~km}$. If the temperature gradient is below about $4 \times 10^{-6}{ }^{\circ} \mathrm{K} \mathrm{cm}^{-1}$, the observed temperatures at $1000 \mathrm{~km}$ are usually greater than $T_{l}\left(\sim 1500^{\circ} \mathrm{K}\right)$. Hence, in that case the flow will always be subsonic.

If $\partial T / \partial r \geq 4 \times 10^{-6}{ }^{\circ} \mathrm{Kcm}^{-1}$, the observed temperatures at $1000 \mathrm{~km}$ are within the range for which a critical point exists at $1000 \mathrm{~km}$ or at higher altitudes. Furthermore, temperature gradients of $10^{-5}{ }^{\circ} \mathrm{Kcm}^{-1}$ and higher are commonly observed at $1000 \mathrm{~km}$ at midlatitudes. Our calculations, therefore, show that the temperature gradient has an important effect in determining the flow characteristics and that supersonic flows in the collapsing early night-time protonosphere cannot be ruled out.

Of course, the fact that a critical point exists in the protonosphere does not imply that the flow will necessarily become supersonic. It may follow any of the solution curves labeled $(A)$ in Fig. 2. Whether or not the flow will actually turn supersonic in a situation where a critical point exists in the protonosphere depends on the boundary value of the Mach number at some upper boundary, say the equatorial plane. If this boundary value equals some critical value, then the flow is described by the critical solution (curve $B$ of Fig. 2). If it is below the critical value (curve $A$ ), the Mach number peaks at an absolute value less than unity. The family of curves of Fig. 2 is understood to apply to the same temperature profile with the different solution curves corresponding to different boundary values of $M$.

Even without supersonic flows it appears that the ion transport speeds are sufficiently high to necessitate the inclusion of the ion inertia term $M \partial M / \partial s$ in calculations of plasma flow. A further difficulty apparent in most calculations of time dependent flows in the topside ionosphere is the neglect of the term $\partial M / \partial t$. In the diffusion approximation the ion momentum equation is written from (2) as

$$
u=-\frac{c}{v_{\mathrm{in}}}\left(\frac{1}{n} \frac{\partial n}{\partial s}+\frac{1}{c^{2}} \frac{\partial \phi}{\partial s}\right),
$$

where both time and space derivatives of the transport speed have been ignored. In Equation (10) the transport speed-profile is assumed to follow instantaneously changes in ion density over the entire field tube. However, we note that the rarefaction and expansion waves associated with changes in the lower boundary pressure can travel upwards only at about the ion sound speed; i.e. $7 \mathrm{~km} \mathrm{sec}^{-1}$ for $\mathrm{H}^{+}$at $3000^{\circ} \mathrm{K}$. Since the distance along the field 
tube to the geomagnetic equator is long at mid-geomagnetic latitudes, $\left(17,000 \mathrm{~km}\right.$ at $50^{\circ}$ geomagnetic latitude), it is apparent that changes in density or velocity in the outer portions of the field tube cannot be expected to rapidly follow the changes at the lower boundary.

\section{CONCLUSION}

In conclusion, it appears that supersonic flow resulting from early night-time collapse of ionization in mid-latitude field tubes is a definite possibility which is presently being investigated by the authors in greater detail. It has been shown that the existence of a nonvanishing temperature gradient has a decisive influence on the location of the critical point, and therefore on the possibility of supersonic flow. Finally, it is noted that theoretical analyses which neglect ion inertia and the time dependence of the plasma transport speed over-estimate the time response of the plasma and will therefore not yield results directly applicable to the topside ionospheric environment during periods of rapidly changing conditions (as post-sunset).

Acknowledgements-The authors wish to acknowledge numerous helpful conversations with A. F. Nagy and W. I. Axford. This research was supported in part, by the National Aeronautics and Space Administration under Grants No. NGR-23-005-015 and NGR-05-009-075.

\section{REFERENCES}

Banks, P. M. (1966). Collision frequencies and energy transfers-Ions. Planet. Space Sci. 14, 1105.

BANKs, P. M., and Holzer, T. E. (1969). Features of plasma transport in the upper atmosphere. J.geophys. Res. 74, 6304.

Brace, L. H., Reddy, B. M. and MaYR, H. G. (1967). Global behavior of the ionosphere at $1000 \mathrm{~km}$ altitude. J. geophys. Res. 72, 265.

NAGY, A. F., BAUER, P. and FONTHEIM, E. G. (1968). Nighttime cooling of the protonosphere. J. geophys. Res. 73, 6259. 